\title{
Host Drosophila fitness and hormonal status depends on the genotype of Wolbachia symbiont
}

\author{
N.E. Gruntenko*, N.V. Adonyeva, Y.Y. Ilinsky, E.V. Burdina, O.V. Andreenkova, \\ R.A. Bykov, I.Yu. Rauschenbach \\ Institute of Cytology and Genetics SB RAS, Novosibirsk, Russia \\ *e-mail:nataly@bionet.nsc.ru
}

Key words: systems biology, aging, hormone metabolism, symbiont, Drosophila

The idea of study presented was to find out if the effect of prokaryotic symbionts Wolbachia pipientis on the host Drosophila fitness and hormonal status depends on the genotype of the bacteria. For this purpose we used five conplastic strains carrying the nuclear background of interbreeded wild type Bi90 line and cytoplasmic backgrounds with different genotype variants of Wolbachia. Bi90 line treated with tetracycline for 3 generations was used as a control group. We demonstrated that only two out of five investigated Wolbachia variants promote changes in Drosophila fitness and metabolism of two hormones, dopamine and juvenile hormone. wMel, wMel2 wMel4 genotypes of Wolbachia do not show any effect on Drosophila stress resistance, the intensity of dopamine metabolism and fecundity, whereas wMelCS genotype increases first two traits and decreases the fecundity in the beginning of the oviposition increasing it later. Young Drosophila females with wMelCS genotype demonstrate the increased level of juvenile hormone metabolism compared to females with $\mathrm{wMel}$ the control ones, while mature females show the opposite pattern, what corresponds well with the changes in their fecundity. The obtained results are quite surprising because genotypes of wMel group predominate in the nature populations all over the world and wMelCS variants are very rare. Especially interesting that pathogenic wMelPop variant of wMelCS Wolbachia genotype increases fecundity and decreases stress resistance, the intensity of dopamine and juvenile hormone metabolism in young females, while increases juvenile hormone metabolism and decreases the fecundity, stress resistance and the dopamine metabolism in mature females. This is also quite unexpected, because the strong negative influence of wMelPop on host metabolism and stress resistance starts much earlier than mass death of flies carrying this pathogenic strain.

Acknowledgements: The study was supported by the RFBR grant No. 16-04-00060 and State Budgeted Project No. 0324-2018-0016. 